\title{
Comunicação
}

[Communication]

\section{Eficácia do extrato aquoso de Azadiracta indica no controle de Boophilus microplus em bovino}

\author{
[Effectiveness of Azadiracta indica watery extract on the control of bovine ticks \\ Boophilus microplus] \\ M. Valente ${ }^{1}$, A. Barranco ${ }^{2}$, A. B. Sellaive-Villaroel ${ }^{2}$ \\ ${ }^{1}$ Instituto Centro de Ensino Tecnológico - Quixeramobim, CE. \\ ${ }^{2}$ Departamento de Zootecnia - UFC \\ Caixa Postal 12167 \\ 60021-970 - Fortaleza, CE
}

Os carrapatos dos ruminantes causam diminuição da produção de leite e carne e sérios danos ao couro (Jonsson et al., 2001). Podem provocar anemia profunda em casos de grandes infestações e são os únicos vetores importantes da babesiose, doença grave causada pelos protozoários intraeritrocitános Babesia bovis e $B$. bigemina (Alonso et al., 1992). Os produtos químicos utilizados no controle desses parasitas têm sido questionados, especialmente após a ocorrência de resistência dos carrapatos a essas substâncias (Li et al., 2003; 2004).

Azadiracta indica A. Juss, conhecida como Neem, é uma Meliaceae originária da Índia e Birmânia, usada há séculos na medicina Ayurvédica indiana (Conrick, 1994; Saibene, 1997; Neemfoundation..., 2003). A azadiractina é um dos compostos do Neem mais estudado e foi classificada como um pesticida de baixo efeito residual (Guerrini e Kriticos, 1998). Além da azadiractina, foram isolados outros 24 princípios ativos no Neem dotados de atividade biológica sobre o comportamento e o crescimento de artrópodes (Schmutterer, 1990; Conrick, 1994). Essa variedade de componentes reduz de modo significativo a ocorrência de fenômenos de tolerância e resistência ao fítoterápico (Mulla e Tianyun, 1999).

Desde a sua introdução no Brasil, em 1984, Azadiracta indica tem demonstrado boa adaptação ao semi-árido, onde vem sendo

Recebido em 30 de agosto de 2005

Aceito em 23 de agosto de 2007

E-mail: zambs9@hotmail.com amplamente cultivada (Organeem..., 2003; O Povo, 2004). Assim, o uso dessa planta no controle dos carrapatos estimularia a sustentabilidade das criações, diminuindo o impacto ambiental e preparando as condições para a implantação da zootecnia biológica.

Este trabalho teve o objetivo de comparar a eficácia do extrato aquoso de folhas frescas de Neem (Azadiracta indica) com a da amabectina no controle de uma infestação por Boophilus microplus.

O experimento foi realizado nos meses de maio e junho de 2004, com 28 animais (10 vacas e 18 novilhas) das raças Pardo-Suíça (19) e Girolanda (9). As vacas foram mantidas estabuladas e as novilhas em piquete, e todas foram alimentadas com forragem fresca, alimento concentrado e sal mineral.

$\mathrm{O}$ extrato aquoso de Neem foi preparado na fazenda, usando $1 \mathrm{~kg}$ de folhas frescas de Neem, misturadas em 5 litros de água à temperatura ambiente. A mistura, deixada em descanso por 12 horas, foi filtrada e envasada em frasco âmbar. O nível de infestação de cada animal foi determinado por amostragem (Heat et al., 1995). $\mathrm{O}$ grupo tratado com Neem recebeu banhos semanais, com 2 litros de extrato aquoso, durante um mês; o outro foi tratado com amabectinal (Abm) tópica, aplicada no dorso, uma única vez no início do experimento. Três coletas foram realizadas a cada 15 dias durante o mês de observação (dia 0, 15 e 30). O delineamento 
experimental empregado foi o fatorial $2 \times 3$, (dois tratamentos e três momentos). Os dados foram processados no programa estatístico SAS (User's..., 1995). Usaram-se os testes $t$ de Student e Kruskal-Wallis para comparação de médias, para níveis máximos de significância $(\mathrm{P}<0,0001)$ (Dash et al., 1988; Sampaio, 2002).

Na Tab. 1 mostra-se a distribuição da infestação por Boophilus microplus nos dois grupos experimentais, durante todo o teste.

Não houve diferença na infestação por parasitas entre os grupos, o que representa um desempenho interessante do extrato aquoso de folhas de Neem. Houve influência do momento da coleta sobre a infestação, para os dois grupos, isto é, os valores foram semelhantes para os dias 0 e 15 após os tratamentos e diferentes dos resultados obtidos no dia 30 , mostrando controle progressivo da infestação similar nos dois grupos. Houve diferença no número de carrapatos em razão da idade, pois observou-se maior infestação nas novilhas. Este resultado está relacionado à resistência adquirida aos carrapatos, que tende a ser maior com o avanço da idade, em razão das muitas vezes a que os animais foram expostos (Roberts, 1968; Wagland, 1975).

Tabela 1. Média e desvio-padrão da infestação de carrapatos tratados com Neem (Azadiracta indica) e abamectina (abm), em função do tempo

\begin{tabular}{lccc}
\hline \multirow{2}{*}{ rrupos } & \multicolumn{3}{c}{ Infestação média } \\
\cline { 2 - 4 } & Dia 0 & Dia 15 & Dia 30 \\
\hline Neem & $112,78 \pm 197,52 \mathrm{a}$ & $62,5 \pm 66,52 \mathrm{a}$ & $7,71 \pm 9,82 \mathrm{~b}$ \\
Abm & $154,64 \pm 194,77 \mathrm{a}$ & $50,5 \pm 48,06 \mathrm{a}$ & $16,0 \pm 23,5 \mathrm{~b}$ \\
\hline
\end{tabular}

Letras diferentes nas colunas ou linhas significam diferenças estatísticas $(\mathrm{P}<0.0001)$

O óleo extraído das sementes de Neem teve sua eficácia demonstrada in vitro contra carrapatos da família Ixodidae (Ndumu et al., 1999). A preparação da solução aquosa de folhas de Neem é ainda mais simples e rápida, podendo ser feita na própria fazenda. $\mathrm{Na}$ forma como foi usada neste teste, a solução aquosa de folhas de Neem pode substituir a amabectina dentro de um programa zootécnico de controle dos carrapatos bovinos no semi-árido.

Palavras-chave: bovino, Azadiracta indica, Neem, Boophilus microplus

\begin{abstract}
This study was carried out with two groups of bovines naturally infected with Boophilus microplus. One group was treated every week during a month with Neem's watery extract and the other group was treated only once (at the test onset) with amabectin pour-on. The results of the Kruskal-Wallis test showed no significant difference $(P<0.0001)$ between both groups (Neem and amabectin) throughout the experiment. However, time of collection [(day0 = day15) $\neq$ day30] and age brackets (cows $<$ heifers) showed significant effect. Neem's watery extract was cheap and easy to prepare, even at farm and could replace amabectin in the control of Boophilus microplus in bovines.
\end{abstract}

Keywords: bovine, Azadiracta indica, Neem, Boophilus microplus

\footnotetext{
${ }^{1}$ Abamectina 1\% - Jofadel Ind. Farmacêutica S/A - Varginha, Brasil
} 


\section{REFERÊNCIAS BIBLIOGRÁFICAS}

ALONSO, M.; ARRELANO-SOTA, C.; CERESER, V.H. Epidemiology of bovine anaplasmosis and babesiosis in Latin America and the Caribbean. Vet. Sei. Tech. Off. Int. Epiz., v.11, p.713-733, 1992.

CONRICK, J. Neem - The ultimate herb. Winter Park, Florida: Hopeful Communication, 1994. $66 \mathrm{p}$.

DASH, K.M.; HALL, E.; BARGER, IA. The role of arithmetic and geometric mean worm egg count in faecal egg count reduction tests and in monitoring strategic drenching programs in sheep. Austr. Vet. J., v.65, p.66-68, 1988.

GUERRINI, V.H.; RRITICOS, C.M. Effects of azadirachtin on Ctenocephalides felis in the dog and the cat. Vet. Parasitol., v.74, p.289-297, 1998.

HEAT, A.C.G.; LAMPKIN, N.; JOWETT, J.H. Evaluation of non-conventional treatments for control of the biting louse (Bovicola ovis) on sheep. Med. Vet. Entomol., v.9, p.407-412, 1995.

JONSSON, N.N.; DAVIS, R.; DE WITT, M. An estimate of the economic effects of cattle rick (Boophilus microplus) infestation on Queensland dairy farms. Austr. Vet. J., v.79, p.826-831, 2001.

LI, A.Y.; DAVEY R.B.; MILLER, R.J.; et al. Detection and characterization of amitraz resistance in the southem cattle tick, Boophilus microplus (Acari: Ixodidae). J. Med. Entomol., v.41, p.193-200, 2004.

LI, A.Y.; DAVEY, R.B.; MILLER, RJ. et al. Resistance to coumaphos and diazinon in Boophilus microplus (Acari: Ixodidae) and evidence for the involvement of an oxidative detoxification mechanism. J. Med. Entomol., v.40, p.482-490, 2003.

MINISTÉRIO da Agricultura e do Abastecimento. Agricultura., 2004. Disponível em $<$ http://www.agricultura.gov.br. $>$. Acessado em 9 set. 2004.
MULLA, S.; TIANYUN, S. Activity and biology effects of neem products against arthropods of medicai and veterinary importance. J. Am. Mosquito Contr. Assoc., v.15, p.133-152, 1999.

NEEMFOUDATION Spearheading the Neem Revolution, 2003. Disponível em $<$ http://neemfoudation.org $>$. Acessado em 9 set. 2004.

NDUMU, PA.; GEORGE, J.B.D.; CHOUDHURY, M.K. Toxicity of Neem Seed Oil (Azadirachta indica) against the larvae of Amblyomma variegatum a three-host tick in cattle. Phytot. Res., v.13, p.532-534, 1999.

O POVO. Os superpoderes do Nim. 15/agosto/2004. Fortaleza-CE, Brasil.

ORGANEEM. Conheça o Neem, 2003. Disponível em <http:// www.organeem.com.br $>$. Acessado em 9 set. 2004.

ROBERTS, J.A., Resistance to cattle to the cattle tick Boophilus microplus (Canestrini, 1987). 1. Development of ticks on Bos taurus. $J$. Parasitol., v.54, p.663-666, 1968.

SAIBENE, A Azadiracta indica, il Neem: tradizione ayurvedica e indagine farmacologica Erborist. Dom., p.61-79, 1997.

SAMPAIO, I.B.M. Estatística aplicada à experimentação animal. 2.ed. Belo Horizonte: FEPMVZ, 2002, 265p.

USER'S Guide: Statistic. Cary, NC: SAS Institute, 1995.

SCHMUTTERER, H. Properties and potencial of nature pesticides from the nim tree Azadirachta indica. Ann. Rev. Entomol., v.35, p.271-297, 1990.

WAGLAND, B.M. Host resistance to cattle tick (Boophilus microplus) in Brahman (Bos indicus) cattle. I. Response of previously unexposed cattle to four infestations with 20.000 larves. Aust. J. Agric. Res., v.26, p.1073-1078,1975. 